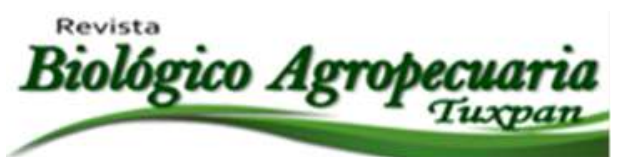

\title{
Validación de características foliares de Laguncularia racemosa ubicada en manglares de Tuxpan, Veracruz
}

\begin{abstract}
Validation of foliar characteristics of Laguncularia racemosa located in the mangroves of Tuxpan,
\end{abstract} Veracruz

González-Sánchez Arianna Rubí ${ }^{1 凶}$, Elorza Martínez Pablo², Segura Castillo Ana Cristina ${ }^{3}$, Castillo Rocha, Doris Guadalupe ${ }^{4}$, Martínez Hernández María de Jesús ${ }^{4}$

\begin{abstract}
${ }^{1}$ Investigador Independiente. ${ }^{2}$ Facultad de Ciencias Biológico Agropecuarias, Universidad Veracruzana. Campus Poza Rica-Tuxpan, carretera Tuxpan-Tampico Kilómetro 7.5, Colonia Universitaria, C.P. 92870 Tuxpan, Ver. ${ }^{3}$ Faculta de Biología, Universidad Veracruzana Campus Xalapa. ${ }^{4}$ Facultad de Ciencias Agrícolas, Universidad Veracruzana. Campus Xalapa.
\end{abstract}

\Autor para correspondencia: glezsan.ar@gmail.com

Recibido: $15 / 09 / 2018$

Aceptado: 15/11/2018

\section{RESUMEN}

La importancia de los ecosistemas de manglar es irrefutable por los innumerables beneficios que brindan en distintos ámbitos; sin embargo, pese al valor que se les atribuye, su tasa anual de deforestación en territorio mexicana es de aproximadamente 2.5\%. En México, la extensión de manglares es de 764,486 hectáreas. Entre las especies que los conforman, se encuentra L. racemosa, una de las principales especies a nivel nacional. Las hojas son los órganos más sensibles de las plantas a la contaminación y sirven como indicadores de alteraciones a condiciones ambientales ya que refleja cambios en las características estomáticas lo cual es relevante por la importancia de los estomas en la homeostasis de las plantas. El objetivo de este estudio fue determinar macro- y micro-características foliares de plantas de L. racemosa en dos zonas del sitio Ramsar 1602, una al norte (deteriorada) y otra al sur (no deteriorada) del Río Tuxpan. Se consideraron 12 individuos al azar de cada localidad para obtener material vegetal. El promedio del área de la hoja fue mayor en la Zona no deteriorada, caso contrario con los valores obtenidos para longitud, ancho, densidad e índice estomático, ya que éstas variables fueron mayores en la zona deteriorada.

Palabras clave: índice estomático, densidad estomática, área foliar, Sitio Ramsar.

\begin{abstract}
La importancia de los ecosistemas de manglar es irrefutable por los innumerables beneficios que brindan en los distintos ámbitos; Sin embargo, la tasa anual de deforestación en el territorio mexicano es de aproximadamente 2.5\%. En México, la extensión de manglares es de 764,486 hectáreas. Entre las especies que se conforman, se encuentra L. racemosa, una de las principales especies a nivel nacional.
\end{abstract}


Las hojas son los órganos más sensibles de las plantas a la misma y los indicadores de las alteraciones a las condiciones ambientales, las condiciones estomáticas lo que es relevante por la importancia de los estomas en la homeostasis de las plantas. El objetivo de este estudio fue determinar macro y microcaracterísticas foliares de L. racemosa en dos zonas del sitio Ramsar 1602, una al norte (deteriorada) y otra al sur (no deteriorada) del río Tuxpan. Se consideraron 12 individuos al azar de cada localidad para obtener material vegetal. El promedio del área de la hoja fue mayor en la zona no deteriorada, las variables fueron mayores en la zona deteriorada

Keywords: Índex, stomatal density, area, Ramsar site.

\section{INTRODUCCIÓN}

Los ecosistemas de manglar son importantes componentes del ecosistema por los innumerables servicios que brindan en distintos ámbitos (Viñals, 2002; CONABIO, 2009; Domínguez-Domínguez et al., 2011). Las especies que conforman dichos ecosistemas tienen características muy peculiares que les permiten colonizar y reproducirse en zonas con alto grado de perturbación, con cobertura más extensa en áreas asociadas a grandes descargas de los ríos (Agráz-Hernández et al., 2006). Además de su importancia ecológica, son complejos y dinámicos como resultado de los eventos meteorológicos a los que están sometidos y por su ubicación geográfica (límites continentales y aguas oceánicas). Sin embargo, pese al valor que se les atribuye en México, su tasa anual de deforestación nacional es de aproximadamente $2.5 \%$ (INE, 2005), teniendo una disminución del área que ha pasado de 775 mil ha en el 2005 a 764 mil 486 ha en 2010 (Rodríguez-Zúñiga., 2013). Entre las especies que los conforman las manglares mexicanas se encuentra Laguncularia racemosa, una de las principales especies a nivel nacional y protegida por su gran importancia por las normas, NOM022-SEMARNAT-2003 y NOM-059-ECOL2001 (SEMARNAT, 2003; 2010). La morfología vegetal es un indicador de estrés abiótico y los manglares no son la excepción. Las plantas del manglar están sometidos a constantes cambios hídricos y salinos, lo cual modifica las características de ellos de acuerdo con los gradientes ambientales donde viven (el relieve, el sustrato, la inundación, la salinidad, las perturbaciones naturales y la contaminación). La hoja de una planta es el órgano más sensible en responder a estos cambios en condiciones ambientales, por lo que refleja alteraciones y cambios en la síntesis de proteínas, la pared celular, el espesor de la cutícula y diferencias estomáticas (Trewavas, 2003). Los estomas son vitales en el mantenimiento de la homeostasis de una planta, por eso es importante de conocer su número, forma, tamaño, apertura y cierre; con estos datos uno puede calcular el índice estomática y la densidad estomática. En conjunto, esta información permite identificar los factores que controlan a estos procesos para evaluar los cambios que resultaron en el estrés de las plantas (Croxdale, 2000; Salas et al., 2001 y Da Matta, 2004; Azcón y Talón, 2008).

Los registros de contaminantes en la zona del Sitio Ramsar 1602 "Manglares y Humedales de Tuxpan" son de agentes genotoxicos para las especies marinas; contaminación de sedimentos (Botello et al., 2005), concentración de metales pesados en especies marinas comestibles de Laguna de Tampamachoco (Godínez-Campos, 2012; Lozada-Flores, 2015; Sánchez-Olivares et al., 2014; Macías-Hernández, 2015), contaminación por agroquímicos e hidrocarburos (Vázquez-Botello y Calva-B., 
1998), materia orgánica, residuos de vísceras de peces y moluscos, y escorrentía de materia fecal de ganadería (Basáñez-Muñoz, 2005). Para el monitoreo del efecto de estas contaminantes sobre los manglares es importante conocer las características estomáticas de cada especie para hacer seguimiento a cualquier cambio, con el fin de localizar cambios y analizar el tipo de agente causal. Con estos datos, podríamos entender mejor la dinámica de este manglar y plantear alternativas correspondientes al caso. Por lo anterior, el objetivo de esta investigación fue realizar una caracterización foliar (superficie foliar, largo y ancho del estoma y densidad e índice estomático [DE e ÍE]) de Laguncularia racemosa en el Sitio Ramsar 1602 "Manglares y Humedales de Tuxpan" en dos lugares, una obviamente perturbado y otro con apariencia como más natural.

\section{MATERIALES Y MÉTODOS}

\section{Área de estudio}

El Sitio Ramsar 1602, ubicado en el norte del estado de Veracruz, México (punto central en las coordenadas $21^{\circ} 00^{\prime} \mathrm{N} 097^{\circ} 21^{\prime} \mathrm{W}$ ), abarca 6,870 ha (Ramsar, 2016). El mangle es el principal tipo de vegetación en el área (INEGI, 2015). Dicha vegetación se alimenta por dos principales cuerpos de agua, Laguna Tampamachoco y Río Tuxpan (Lara-Domínguez et al., 2009); este último divide al Sitio Ramsar en dos zonas ubicadas al norte y sur del mismo. LaraDomínguez et al., 2009).

\section{Selección del área de muestreo y toma de datos}

Se seleccionaron dos zonas de muestreo zona (zona norte y zona sur) dentro del Sitio Ramsar en acuerdo con su apariencia de contaminación. La época de muestreo se inició en junio y finalizó en agosto del 2014. Se establecieron el periodo de muestra bajo la premisa que las variables morfológicas que se evaluaron no son cambiantes a corto plazo.

Los datos se tomaron de las hojas al azar de 12 árboles de Laguncularia racemosa por zona (zona norte y zona sur); se recolectaron 60 hojas maduras ubicadas a una altura media en el dosel con exposición lumínica intermedia. Para identificar las especies de los árboles se utilizó la guía de campo de manglares de México de Agráz-Hernández et al. (2006). Se midió el área en $\mathrm{cm}^{2}$ (CI-202 Area meter cid., Inc.) y para la densidad e índice estomática se realizaron impresiones estomáticas con la técnica de microrelieve utilizando cianoacrilato (Weyers y Meidner, 1990). Las impresiones se observaron con un microscopio óptico a $40 \mathrm{X}$ y se digitalizaron cinco fotografías de cinco campos diferentes de cada lámina foliar. Se contabilizó el número de estomas y células epidérmicas a través de las fotografías utilizando el software UTHSCSA Image Tool versión 3.00; una vez contabilizadas las células, se aplicó la fórmula sugerida por Wilkinson (1979) para el cálculo del índice estomático: La DE fue determinado obteniendo el área bajo el objetivo de 40X del microscopio (Lieder, modelo MC-420) $\left(0.159 \mathrm{~mm}^{2}\right)$ y contando en número de estomas $\mathrm{y}$ células por unidad de área; el largo y ancho de los estomas se medió con el software UTHSCSA Image Tool versión 3.00. El análisis estadístico partió de la comprobación de que los datos cumplían con la normalidad, posteriormente se realizó un ANOVA con un nivel de significación $\mathrm{P} \leq 0.05$, y una prueba de Tukey. El programa estadístico utilizado fue SPSS (versión 12.0 1S, SPSS Inc.).

\section{RESULTADOS}

Macromorfología: Las hojas de Laguncularia racemosa son elípticas y redondeadas, tanto en la base como en el ápice, se encuentran en posiciones opuestas. La superficie adaxial tiene 
color verde oscuro brillante y la abaxial, verde amarillento. Los laterales del peciolo poseen dos glándulas secretoras y sobre la superficie abaxial presenta varias glándulas hundidas (Fig. 1). El tipo de hojas, de acuerdo a la ubicación de los estomas, es anfiestomática y el estoma es anomocítico o ranunculáceo (sin células anexas) (Fig. 1). El área foliar promedio fue de $16.9 \mathrm{~cm}^{2}$ para la zona deteriorada y $28.2 \mathrm{~cm}^{2}$ para la zona no deteriorada, una diferencia altamente significativa $(\mathrm{P} \leq 0.001)$.

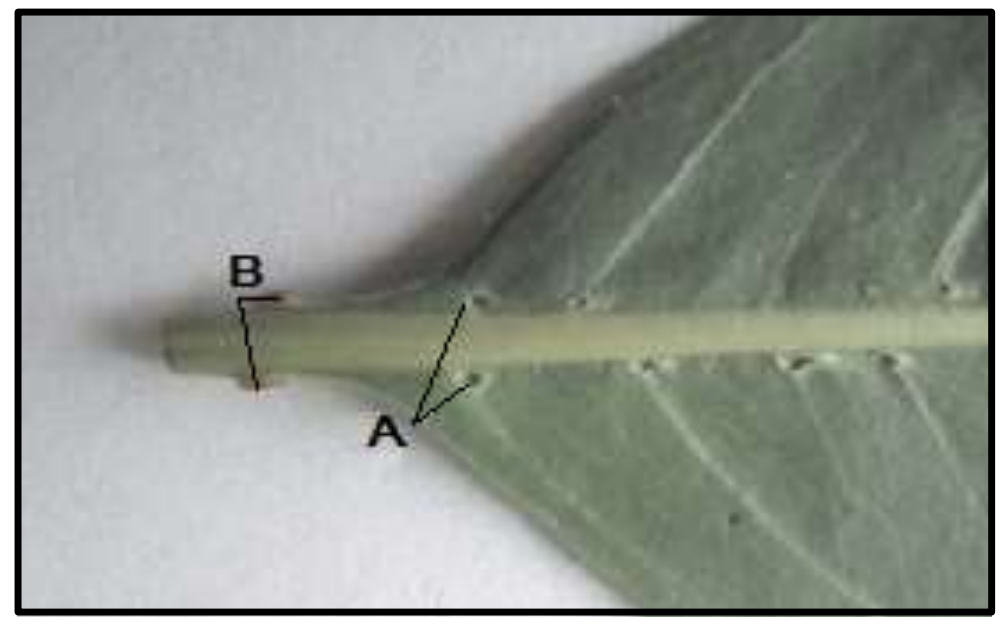

Figura 1 .Hoja anfiestomática de Laguncularia racemosa con glándulas secretoras en los laterales del peciolo (B) y sobre la superficie abaxial presenta varias glándulas hundidas (A).

Micromorfología: El promedio de la densidad estomática adaxial fue 117 estomas $/ \mathrm{mm}^{2}$ para la $\mathrm{ZN}$ y de 78 estomas $/ \mathrm{mm}^{2}$ para la ZS; para la superficie abaxial en la $\mathrm{ZN}$ presentó 73 y en $\mathrm{ZS}$ de 50 estomas $/ \mathrm{mm}^{2}$. El Í. E. (Índice estomático) en la zona deteriorada adaxial fue de $6.20 \%$ promedio y para la superficie abaxial fue de $4.55 \%$, y en la zona no deteriorada adaxial fue de 6.15 y para la superficie abaxial fue de $4.73 \%$ con respecto para la longitud de los estomas en la zona deteriorada adaxial tuvo un promedio de $0.23 \mathrm{~mm}$ y en la zona no deteriorada de $0.11 \mathrm{~mm}$, en cuanto a la longitud para la superficie abaxial se obtuvo $0.22 \mathrm{~mm}$ para la zona deteriorada y $0.12 \mathrm{~mm}$ para la zona no deteriorada. Finalmente el ancho del estoma tuvo un promedio de 0.082 zona deteriorada adaxial y para la superficie abaxial de $0.074 \mathrm{~mm}$ y en la zona no deteriorada adaxial obtuvo 0.052 y para la superficie abaxial de $0.73 \mathrm{~mm}$ (Fig. 2). 


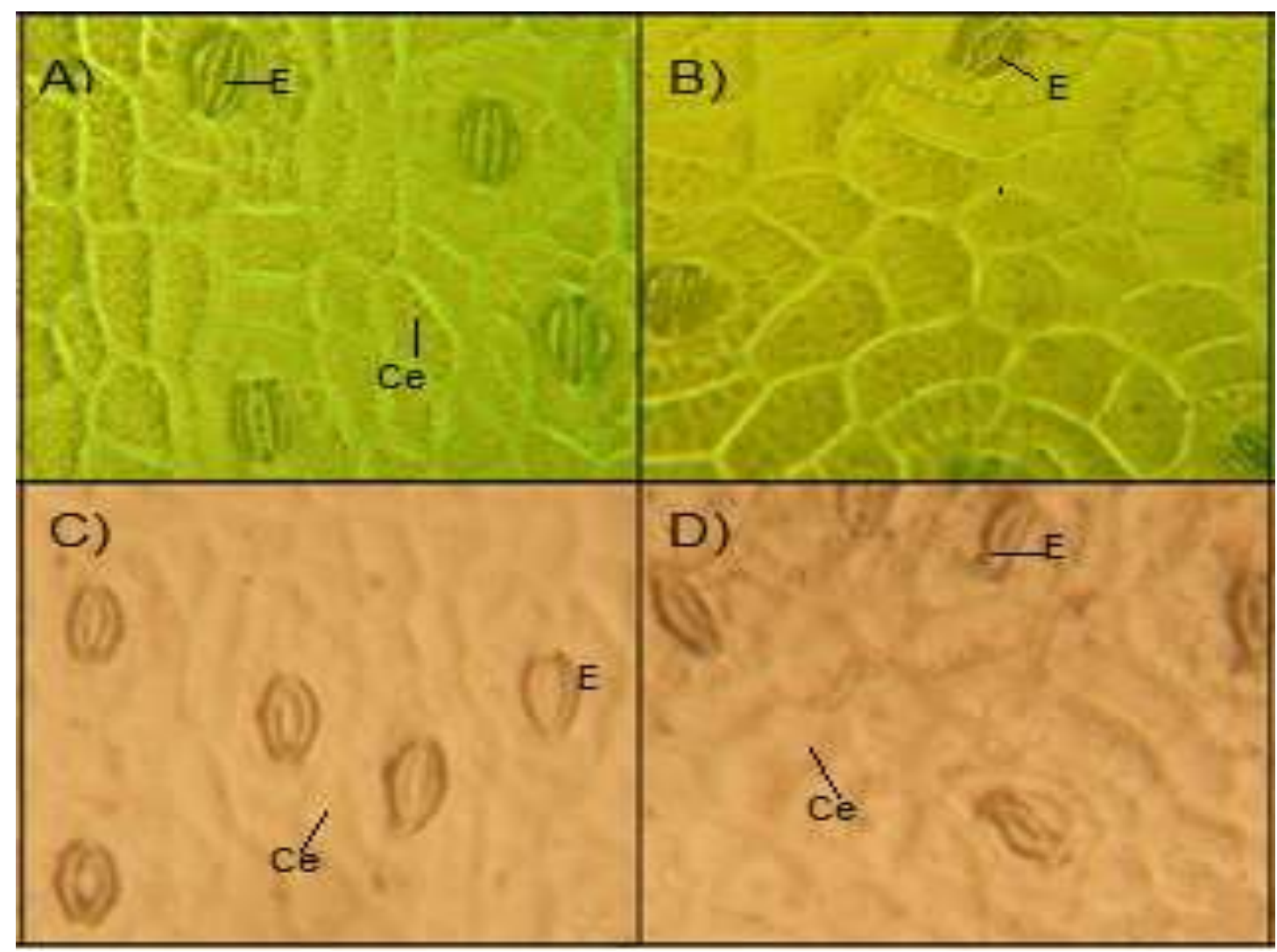

Figura 2. Micro relieve de hojas de Laguncularia racemosa mostrando estomas anomocítico o ranunculáceo ( $\sin$ células anexas) en las superficies adaxial y abaxial de ambas zonas de muestreo. A= superficie adaxial zona sur, $\mathrm{B}=$ superficie abaxial zona sur, $\mathrm{C}=$ superficie adaxial zona norte, $\mathrm{D}=$ superficie abaxial zona norte, $\mathrm{E}=$ Estomas, $\mathrm{Ce}=$ células epidérmicas.

\section{DISCUSIÓN}

La caracterización de la forma y tipo de hoja fue descrita por Agráz-Hernández et al. (2006) en México, Tribaldos (2008) en Panamá, Francisco et al. (2009) en Venezuela, y Díaz et al. (2010) en Cartagena de Indias, Colombia quienes describieron la hoja de Laguncularia racemosa como simple, sin estípulas, en colocación opuestas, forma elípticas a oblongoelípticas, pecíolo generalmente de color rojizo con un par de glándulas en el extremo apical llamados nectarios extraflorales, lo cual coincidieron con nuestros reportes para la misma especie en los Manglares y Humedales de Tuxpan, Veracruz en 2014. Con respecto a la ubicación de los estomas Sereneski-de Lima et al. (2013) en el sur de Brasil, Rodríguez-
Rodríguez et al. (2011) en el Caribe Insular colombiano, Pelozo $(2012,2015)$ en las Bahías de Antonina y Guaratuba, Brasil reportaron la lámina foliar de Laguncularia racemosa como anfiestomáticas, lo cual concuerda con nuestro reporte al encontrar estomas en ambas superficies de la hoja, en la misma especie en Manglares de Tuxpan, Veracruz en el 2014.

El tipo de estoma reportado por Pelozo et al. (2012, 2015) en las Bahías de Antonina y Guaratuba en, Brasil fue anomocítico o, ranunculáceo, es decir estomas sin células anexas en la especie de Laguncularia racemosa tal y como se reporta en este estudio para manglares de Tuxpan Veracruz.

En cuanto al área foliar Sobrado (2007) reportó en plantas cultivadas en invernadero bajo diferente concentración de salinidad en 
Venezuela áreas de 21, 22 y $25 \mathrm{~cm}^{2}$; Lugo et al. (2007) reportando $20.2 \mathrm{~cm}^{2}$ en plantas de la bahía de Jobos, Puerto Rico; Tavares et al, (2009) obtuvieron un área de $24.9 \mathrm{~cm}^{2}$ para plantas recolectadas cerca de la Bahía de Sepetiba frontera sur de la y Reserva Arqueológica y Biológica Guaratuba, Río de Janeiro, Brasil; Lira-Medeiros et al. (2010) en la Bahía de Sepetiba, Brasil ubicaron dos zonas de estudio teniendo en promedio 23 a $25 \mathrm{~cm}^{2}$ para el área de la hoja de Laguncularia racemosa; Sereneski-de Lima et al. (2013) en manglares de Brasil obtuvieron área de $26.4 \mathrm{~cm}^{2}$. Lo anterior no difiere con lo encontrado en este estudio, ya que se obtuvo un promedio de $16.9 \mathrm{~cm}^{2}$ para la zona deteriorada y $28.2 \mathrm{~cm}^{2}$ para la zona no deteriorada.

La densidad estomática de Laguncularia racemosa se tiene el antecedente de RodríguezRodríguez et al. (2011) para la superficie adaxial una densidad de 84 a 94 estomas $/ \mathrm{mm}^{2}$ y en la abaxial de 61 a 76 estomas $/ \mathrm{mm}^{2}$ en manglares de San Andrés, Colombia y de Seshavatharan y Srivalli (1989) en manglares de la India quienes registraron 170 para superficie adaxial y 100 estomas $/ \mathrm{mm}^{2}$ para la superficie abaxial. Mientras que este estudio la superficie adaxial mostró una densidad de 117 en la zona deteriorada y 78 estomas $/ \mathrm{mm}^{2}$ para la zona no deteriorad y para la superficie abaxial la densidad vario de 73 en la zona deteriorada y 50 estomas $/ \mathrm{mm}^{2}$ en la no deteriorada del sitio Ramsar 1602 lo que nos demuestra que se obtuvieron diferencias entre el número de estomas para los tres estudios, en los manglares de Colombia la densidad fue un poco mayor a la reportada en esta investigación, pero lo que corresponde a la India la densidad es mayor casi en un $100 \%$, otra característica que sobresale es que siempre es mayor la densidad en la superficie adaxial en comparación con la abaxial.
El índice estomático reportado por Seshavatharan y Srivalli (1989) en manglares del estuario de Godavari, área cercana de Balusu Tippa y Coringa en la India para Lumnitzera racemosa fue de 13.2 para la superficie adaxial y para la adaxial de 9.2 lo cual es mayor en comparación a lo obtenido en este estudio ya que para la zona deteriorada en la superficie adaxial fue de 6.19 y para la superficie abaxial fue de $4.55 \%$, en la zona no deteriorada superficie adaxial fue de 6.15 y para la superficie abaxial fue de $4.73 \%$, en este caso el índice estomático fue mayor en los manglares de la India, pero el comportamiento de mayor índice en la superficie adaxial con respecto a la abaxial se mantuvo en ambos estudios.

El tamaño de los estomas también fue analizado por Rodríguez- Rodríguez et al. (2011) en manglares colombianos en la misma especie obteniendo la longitud de 0.039 a 0.043 en la superficie adaxial y $0.034 \mathrm{~mm}$ a $0.041 \mathrm{~mm}$ en la abaxial. Seshavatharan y Srivalli (1989) informaron para una especie de manglar con diferente género Lumnitzera racemosa en la India una longitud abaxial de $0.0253 \mathrm{~mm}$ y $0.0256 \mathrm{~mm}$ para la superficie adaxial, mientras que en este estudio se obtuvo una longitud de $0.23 \mathrm{~mm}$ para la superficie adaxial y $0.22 \mathrm{~mm}$ en la abaxial en la zona deteriorada y $0.11 \mathrm{~mm}$ adaxial y $0.12 \mathrm{~mm}$ para la abaxial de la zona no deteriorada. Seshavatharan y Srivalli (1989) indicaron igualmente el ancho del estoma para la especie de Lumnitzera racemosa en la India con $0.018 \mathrm{~mm}$ abaxial y $0.0156 \mathrm{~mm}$ para la superficie adaxial, en tanto en este estudio se obtuvo $0.082 \mathrm{~mm}$ para la superficie adaxial y $0.074 \mathrm{~mm}$ en la abaxial en la zona deteriorada; $y$ $0.052 \mathrm{~mm}$ adaxial y $0.0732 \mathrm{~mm}$ para la abaxial de la zona no deteriorada. En este caso el tamaño de los estomas de la India y Colombia fue menor lo que contribuye a que se encuentre un mayor índice estomático en esas zonas. 
Las variaciones en las macro- y microcaracterísticas foliares de Laguncularia racemosa, menor desarrollo del área foliar, el aumento en el largo y ancho estomático en la zona deteriorada puede asociarse a diversos factores como restricciones ambientales de su hábitat o a las condiciones de epigenética de la especie, tal como lo menciona Lira-Medeiros et al. (2010), otra posible explicación son las afectaciones antropogénicas a las que la zona está sometida constantemente como lo han documentado varios autores, Macías-Hernández (2015), Sánchez-Olivares et al. (2014) y Mendoza-Díaz (2010) reportaron presencia de cadmio, cromo y plomo cercanos al área deteriorada;

Botello et al. (2005) reportaron la zona costera de Tuxpan afectada por hidrocarburos disueltos para aguas superficiales no contaminadas; (Basáñez, 2005) reporta presencia de agroquímicos, materia orgánica, residuos de vísceras de especies marinas y escorrentía de materia fecal de ganadería; Pezeshki et al. (1990) Mencionan reportes de disminución del área foliar de Laguncularia racemosa como respuesta a las inundaciones por concepto de menor salinidad en el medio. Sin embargo, pese a los reportes que se tienen, no se puede saber en concreto cuales son los agentes causales que modifican la morfología foliar de Laguncularia racemosa por lo que se debe continuar con ésta línea de investigación para tener la certeza, de cuál o cuáles de los diversos contaminantes registrados en la zona, son el que afecta el desarrollo de las plantas.

Esta investigación y sus resultados fortalecen la necesidad de continuar indagando sobre los agentes causales que modifican la estructura del Sitio Ramsar 1602 y así, favorecer su conservación desde una perspectiva integral y no aislada. Conocer la contaminación en áreas protegidas favorece entre otras cosas a dar mejor manejo a zonas afectadas para comprender el escaso desarrollo de las especies que había en ellas, principalmente las especies de manglar.

\section{CONCLUSIONES}

La respuesta morfológica a nivel estomático como aumento de la densidad y tamaño puede deberse a mecanismos de regulación de la especie ante los factores que prevalecen en esa zona en particular. Las diferencias en las micro y macro características son evidentes, por lo que se requiere continuar en el futuro con estudios que contemplen la cuantificación de variables como potencial hídrico foliar y del suelo, así como de hidrocarburos de diferente fracción, aspectos fisicoquímicos del suelo y agua a largo plazo.

\section{LITERATURA CITADA}

Agráz-Hernández; Noriega-Trejo, R.; LópezPortillo, J.; Flores-Verdugo, F.J.; JiménezZacarías, J.J., 2006. Guía de Campo. Identificación de los Manglares en México. Universidad Autónoma de Campeche. 45 p.

Azcón-Bieto, J. y M. Talón. Fundamentos de la Fisiología Vegetal. 2da Ed. Mc Graw Hill. Barcelona, España.656 p.

Botello, A. V., J. Rendón-Von Osten, G. GoldBouchot y C. Agraz-Hernández (Eds.). 2005. Golfo de México Contaminación e Impacto Ambiental: Diagnóstico y Tendencias, 2da Edición. Universidad Autónoma de Campeche, Universidad Nacional Autónoma de México, y Instituto Nacional de Ecología. 696 p.

Comisión Nacional para el Conocimiento y Uso de la Biodiversidad. 2009. Sitios de manglar con relevancia biológica y con 
necesidades de rehabilitación ecológica. CONABIO, México, D.F.

Croxdale, J. 2000. Stomatal patterning in angiosperms. American Journal of Botany 87 (8):

1069-1080. https://doi.org/10.2307/2656643

Da Matta, F. 2004. Exploring droght tolerante in coffe a physiological approach with some insigths for plants breeding. Brazilian Journal Plant physiology. 16 (1): 1-6. https://doi.org/10.1590/S1677-042020040 00100001

Díaz, M. C., Castro, A. I., Manjarrez, P. G. 2010. Manglares de Cartagena de Indias. Patrimonio Biológico y Fuente de Biodiversidad. Fundación Universitaria Tecnológico Comfenalco. Cartagena, Colombia. $72 \mathrm{p}$.

Domínguez-Domínguez M., J. Zavala-Cruz, P. Martínez-Zurimendi. 2011. Manejo forestal sustentable de los manglares de Tabasco. Secretaría de Recursos Naturales y Protección Ambiental. Colegio de Postgraduados. Villahermosa, Tabasco, México. 137 p.

Francisco, M., Díaz, M. y Sánchez-Misle, F. 2009. Descripción morfoanatómica de los tipos de glándulas foliares en el mangle blanco Laguncularia racemosa 1. gaertn (f.). Acta Microscopica. 18:237-252.

Instituto Nacional de Ecología. 2005. Evaluación preliminar de las tasas de pérdida de superficie de manglar en México. Secretaria de Medio Ambiente y Recursos Naturales. Dirección General de Investigación de Ordenamiento Ecológico y Conservación de los Ecosistemas. México $21 \mathrm{p}$.

Instituto Nacional de Estadística y Geografía. 2015. Guía para la interpretación de cartografía: uso del suelo y vegetación: escala 1:250, 000: serie V / Instituto Nacional de Estadística y Geografía. México. 195 p.

Lara-Domínguez, A. L.; J. López-Portillo; A. Ávila-Ángeles y A. D. Vázquez-Lule. Caracterización del sitio de manglar. Tuxpan, en Comisión Nacional para el Conocimiento y Uso de la Biodiversidad (CONABIO). 2009. Sitios de manglar con relevancia biológica y con necesidades de rehabilitación ecológica. CONABIO, México, D.F. 6 p.

Lira-Medeiros, C. F., Parisod, C, Fernandes, R. A., Mata, C. S., Cardoso, M. A. 2010 Epigenetic Variation in Mangrove Plants Occurring in Contrasting Natural Environment. PLoS ONE 5(4): e10326. https://doi.org/10.1371/journal.pone.0010 326

Lugo, A. E., Medina, E., Cuevas, E., Citrón, G., Laboy, N. E. N. y Schäeffer N. Y. 2007 Ecophysiology of a Mangrove Forest in Jobos Bay, Puerto Rico. Caribbean Journal of Science 43: 200-219 https://doi.org/10.18475/cjos.v43i2.a6

Macías-Hernández, P. G. 2015. Determinación de metales pesados $(\mathrm{Pb}, \mathrm{Cd}, \mathrm{Cr})$ en agua y sedimentos de la zona estuarina del río Tuxpan, Veracruz. Tesis de Maestría en Ciencias del Ambiente, Universidad Veracruzana. $53 \mathrm{p}$.

Mendoza-Díaz，F. 2010. Determinación de metales pesados $\mathrm{Cd}, \mathrm{Cr}, \mathrm{Cu}$ y $\mathrm{Pb}$ en Farfantepenaeus aztecus (Ives, 1981) colectados en la laguna de Tampamachoco, Veracruz. Tesis de Maestría en manejo de ecosistemas marinos y costeros, Universidad Veracruzana. $80 \mathrm{p}$. 
Pelozo, A. 2012. Regeneração natural das espécies arbóreas dos manguezais do paraná: estrutura e morfología funcional. Tesis doctorado. Botânica, Área de concentração em Estrutura e Fisiologia do Desenvolvimento Vegetal, Departamento de Botânica, Setor de Ciências Biológicas da Universidade Federal do Paraná, Brasil. $63 p$.

Pelozo, A., Boeger, T. M.G., Sereneski-Lima, C., Soffiatti, P. 2015. Leaf morphological strategies of seedlings and saplings of Rhizophora mangle (Rhizophoraceae), Laguncularia racemosa (Combretaceae) and Avicennia schaueriana (Acanthaceae) from Southern Brazil. Revista de Biología Tropical No1. https://doi.org/10.15517/rbt.v64i1.17923

Pezeshki, S. R., DeLaune, R. D., Patrick, W. H. 1990. Differential response of selected mangroves to soil flooding and salinity: gas exchange and biomass partitioning. Canadian Journal of Forest Research, 1990, 20(7):869-874. https://doi.org/10.1139/x90-116

Ramsar. 2016. The List of Wetlands of International Importance. Página en Red: www.ramsar.org; (Consultada 22 de febrero 2016).

Rodríguez-Rodríguez, J. A., Mancera-Pineda, J.E; Melgarejo, L.M. 2011. Cambios en conductancia y morfología estomática en manglares del Caribe que habitan condiciones contrastantes de salinidad. Trabajo de Grado para optar al título de Bióloga. Universidad Nacional de Colombia.

Rodríguez-Zúñiga, M.T., Troche-Souza C., Vázquez-Lule, A. D., Márquez-Mendoza, J. D., Vázquez- Balderas, B., ValderramaLanderos, L., Velázquez-Salazar, S., Cruz-
López, M. I., Ressl, R., Uribe-Martínez, A., Cerdeira-Estrada, S., Acosta Velázquez, J., Díaz-Gallegos, J., JiménezRosenberg, R., Fueyo Mac Donald, L. y Galindo-Leal, C. 2013. Manglares de México/ Extensión, distribución y monitoreo. Comisión Nacional para el Conocimiento y Uso de la Biodiversidad. México D.F. 128 pp.

Salas, J., M. Sanabria y R. Pire. 2001. Variación en el índice y densidad estomática en plantas de tomate (Lycopersicon esculetum Mill) Sometidas a tratamientos salinos. Bioagro 13 (3). 99-104 p.

Sánchez-Olivares, M. A., M. A. LópezJiménez, M. López-Ortega y Vázquez-L. Castán. 2014. Concentración de Cadmio (Cd) en Callinectes sapidus (Decapoda: Portunidae) en la Laguna de Tampamachoco, Veracruz, México. Revista Científica Biológico Agropecuaria Tuxpan 2(4): 807-815.

SEMARNAT; Secretaria de Medio Ambiente y Recursos Naturales. 2003. NOM-022SEMARNAT-2003, Norma que establece las especificaciones para la preservación, conservación, aprovechamiento sustentable y restauración de los humedales costeros en zonas de manglar. Diarios Oficial de la Federación. Ultima reforma publicada el 10 de abril de 2003, p. 26-47.

SEMARNAT; Secretaria de Medio Ambiente y Recursos Naturales. 2010. NOM-059SEMARNAT-2010, Norma que establece la protección ambiental- Especies nativas de México de flora y fauna silvestreCategorías de riesgo y especificaciones para su inclusión o cambio-Lista de especies en riesgo. Diarios Oficial de la 
Federación. Ultima reforma publicada el 30 de diciembre de 2010, p. 1-77.

Sereneski-de Lima, C., Torres-Boeger, R. M., Larcher-de Carvalho, L., Pelozzo and Soffiatti P. 2013. Sclerophylly in mangrove tree species from South Brazil. Revista Mexicana de Biodiversidad 84: 1159-1166. https://doi.org/10.7550/rmb.32149

Seshavatharam y Srivalli, M. 1989. Systematic leaf anatomy of some Indian mangroves. Proc. Indian Acad. Sci. (Plant Sci.). 99: 557-565.

Sobrado, M. A. 2007. Relationship of water transport to anatomical features in the mangrove Laguncularia racemosa grown under contrasting salinities. New Phytologist, 173: 584-591. https://doi.org/10.1111/j.1469-8137.2006. 01927.x

Tavares, D. M. L. F. y Luna P. A. 2009. Leaf damage in a mangrove swamp at Sepetiba Bay, Rio de Janeiro, Brazil. Revista Brasil. Bot., V.32, n.4, p.715-724 https://doi.org/10.1590/S0100-840420 09000400010
Trewavas, A. 2003. Aspects of Plant Intelligence. Annals of Botany 92: 1-20. https://doi.org/10.1093/aob/mcg101

Tribaldos T., Ángel. 2008. Guía de identificación de mangles del humedal Bahía de Panamá.Proyecto de Biomonitoreo Participativo en el Humedal Bahía de Panamá.Sociedad Audubon de Panamá,Panamá.

Viñals, M. J. (coord.) 2002. El patrimonio cultural de los humedales. Ministerio de Medio Ambiente. p. 272.

Weyer, J., Meidner, H. 1990. Methods en stomatal research. Longman, Scientific \& Technical. p: 223.

Wilkinson H. 1979. The plant surface (mainly leaf). In Metcalfe C. R y L. Chalk eds. Anatomy of Dicotyledons. Oxford, London, UK. Claredon Press. p: 97-165.

Copyright (c) 2018 Arianna Rubi González Sánchez, Pablo Elorza Martinez, Ana Cristina S egura Castillo, Doris Guadalupe Castillo Rocha y Maria de Jesús Martinez Hemández

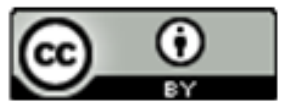

E ste tex to está protegido por una licencia CreativeCommons 4.0 .

Usted es libre para Compartir —copiar y redistribuir el $\mathrm{m}$ aterial en cualquier medio o formato-y Adaptar el documento —remezclar, transformar y crear a partir del material- para cualquier propósito, incluso para fines comerciales, siempre que cumpla la condición de:

Atribución: Usted debe dar crédito a la obra original de manera adecuada, proporcionar un enlace a la licencia, e in dicar si se han realizado cambios. Puede hacerlo en cualquier forma razonable, pero no de forma tal que sugiera que tiene el apoyo del licenciante o 10 recibe por el uso que hace de la obra.

Resumendelicencia - Textocompletodelalicencia 